\title{
MYOFASCIAL PAIN TREATMENT; LOCAL ANESTHESIA INJECTION VERSUS CORTICOSTEROIDS. (RAMDOMIZED CONTROLLED CLINICAL TRIAL)
}

\author{
Omniya M Abdel Aziz* and Hesham S. Abdelmoneim*
}

\begin{abstract}
Myofascial trigger points are one of the most common etiologies of orofacial pain. Although being quite simple to diagnose and easily detected it is eventually a difficult disease that poses a challenge for clinicians. The aim of this study is to evaluate the benefits of combining corticosteroids with local anaesthesia in management of myofascial trigger points. This study was conducted on 30 patients randomly allocated into either the control or the intervention groups. The results of the primary outcome stated an improvement in the pain score for both groups without statistical difference, and the secondary outcome reported an improvement of the MMO for both groups with better results for the intervention over the control groups despite also falling to be statistically nonsignificant. Concluding that Corticosteroid adjunction with local anaesthesia resulted in better pain and MMO results than the use of LA alone for the management of MTrPs.
\end{abstract}

Keywords; myofascila trigger points, MTrPs needling, Cortocosteroids trigger point injection

\section{INTRODUCTION}

Myofascial trigger points are one of the most common causes of orofacial pain. Although being simple to diagnose and easily detect noninflammatory form of myalgia, it is eventually a difficult disease that's posing a challenge for clinicians specialized in orofacial pain in order to locate the etiological factor, in addition to the exact pain source, not forgetting the challenge faced to manage it. ${ }^{(1)}$
Due to the difficulty in recognizing the predisposing factor of that disease and as considered to be multifactorial in nature, myofascial pain (MP) and myofascial trigger points (MTrPs) are yielded to be a difficult pathology that needs a lot of experience and knowledge to successfully manage it. Among the most commonly noted causes for MP are malocclusion, trauma, mechanical stresses and imbalance, temporomandibular joint disorders, sleep disturbance and vitamins insufficiencies..$^{(2,3)}$

*Lecturer of Oral and Maxillofacial Surgery, Faculty of Dentistry, Cairo University. 
An array of perpetual factors has been described by Travell and Simons deducting nutritional, metabolic, mechanical and psychological categories, where bruxism, malocclusion, joint hypermobility, TMJ internal derangement, ergonomic stress and poor postural mechanics were considered to be the most notable mechanical factors. ${ }^{(6)}$

Managing MP is usually frustrating and hard and depends to a huge extent on clinicians experience because treating the underlying etiology is the ideal strategy for MP successful therapy. Failure to successfully manage the etiology usually results in reactivation of MTrPs and persistence of MP symptoms. Since being multifactorial in nature so the treatment has to be individualized and tailored to each case in order to properly target its etiological precipitants. Among the most commonly proposed treatment options are behavioral modification, physical therapy, medications, and needling therapy and/or a combination of them. Failing to document a gold standard treatment modality advocated treating clinicians to implement a multifaceted approach involving combinations of two or more treatment modalities. ${ }^{(3)}$

Myofascial trigger point (MTrP) has been reported as a hyperirritable spot in a skeletal muscle that is usually allocated within a hypersensitive palpable node along a muscle taut band. Active trigger points usually prevail in tension-type headaches, which are coherent with the hypothesis dictating that peripheral mechanisms are commonly involved in the pathophysiology of this headache disorder category. It has been previously reported that myofascial trigger points are prevalent in both migraine and tension-type headache, however their role in the pathophysiology of these disorders is unclarified. ${ }^{(4)}$

As the diagnosis of myofascial pain disorder is utterly based on exclusion of relevant temporomandibular disorders (TMD), trigger points identification in the taut band is through palpation of these sensitive nodules, local twitch response may result in addition to specific patterns of referred pain could be associated with each trigger point. Among the management techniques are trigger point inactivation via dry needling, local anaesthesia injection, botox injection, corticosteroids injection, occlusal splint therapy, patient education, behavior therapy, physiotherapy, drug therapy and any combinations. Drugs utilized to manage MPD include non-steroidal anti-inflammatory drugs (NSAIDS), analgesics, muscle relaxants and antidepressants. ${ }^{(5-8)}$

Myofascial trigger point injection resembles one of the most widely reported effective therapeutic approaches to manage myofascial pain and thereby manage chronic (active) trigger points through fibrotic scar formation. Several postulations have been made about the mechanisms involved in the inactivation of a trigger point ${ }^{(9)}$ :

- Mechanical disruption of the muscles fibers and the nerve endings.

- Mechanical disruption of muscle fibers, leading to elevated extracellular potassium levels which in turn result in nerves depolarization.

- Pain positive feedback interruption.

- Local dilution of nociceptive substances by the local anesthetic or saline infiltrates injected to the area.

- Local anesthetic agents vasodilatory effect which in turn result in rapid removal of toxic metabolites.

An array of substances could be injected as local anaesthetics (procaine, lidocaine, mepivacaine), saline solution, corticosteroids and botulinum toxin. Dry-needling which was described as intramuscular mechanical stimulation technique carried out using a fine, solid needle without any solution to be administered (injected), in order to cause disruption of muscle fibers and nerve endings, also resulted in satisfactory outcomes. ${ }^{(10)}$ 
It has been reported that local anesthesia relieved the patients pain according to the visual analog scale (VAS), it has been reported to be statistically non significantly difference from other needling techniques despite being with promising results. ${ }^{(11)}$ and as previously documented that dry needling in MTrPs in the pterygoid muscles can be an effective modality in the management of patients with myofascial pain. ${ }^{(12)}$

Corticosteroids has also been described in literature for management of MTrPs due to their potent anti-inflamatory action and are known to affect glucose/protein metabolism, Control rate of mRNA synthesis, protein synthesis and Suppress immunological response of lymphocytes. As corticosteroids injection in soft tissue usually results in post-inj. pain flare (2-10\%), so they were usually combined with anaesthetics to reduce the injection pain flare and make the procedure more acceptable. ${ }^{(13)}$

As previously stated in literature and due to the lack of gold standard in trigger points management therefor the aim of our study was to evaluate the benefit of using corticosteroid injections in the management of myofascial pain trigger points.

\section{PATIENTS AND METHODS}

Patients with active MTrPs within the jaw muscles were randomly allocated to one of two study arms with 1:1 allocation ratio via computerized sequence generation: the 'intervention' group (B) who underwent trigger point injection with corticosteroids (dexamethasone $0.2 \mathrm{ml}$ with $0.2 \mathrm{ml}$ plain mepivicaine local anaesthetic) or the control group (A) who underwent LA injection (3\% mepivicaine without vasoconstrictor) of the trigger points. This study was conducted on 30 patients selected from the out-patient clinic, Department of Oral and Maxillofacial Surgery, Faculty of Dentistry, Cairo University. Patients enrolled were randomly allocated into either group A or B so that each group included 15 cases.

\section{Inclusion/exclusion criteria}

Patients were eligible if they had had spontaneous orofacial pain of muscular origin of more than 6 weeks. Excluded from the study patients who were using any medication to reduce the pain and/or have any effect on the skeletal muscle; were receiving physical therapy or wearing occlusal splint in the same period of the research; were pregnant and / or with medical problems that may interfere with the procedures such as bleeding disorders or allergy to LA solutions or cognitive impairment.

\section{Interventions}

Localization of the MTrPs starts with identifying taut band by palpating perpendicular to the muscle fibres direction ${ }^{21}$. The most symptomatic site along the taut bands were considered to be MTrPs.

Once a $\operatorname{TrP}$ was identified, the index finger was slided over the taut band to stabilize it at the fingertip. The overlying skin has been cleansed with alcohol (Alcohol swap: one pad saturated with 70\% Isopropyl Alcohol.pharmapack Pharmaceuticals industries Co., Giza, Egypt). Aspirating dental syringes and 27 gauge needles were used to inject the MTrPs. The needle was introduced into theMTrPs just opposite to fingertip. As the needle contacted MTrP, it induced pain and/or local twitch response.One fourth of the carpule $(\sim 0.5 \mathrm{ml})$ of $3 \%$ mepivacaine (Mepecaine L: Mepecaine Hcl 3\%, Alexandria Co. for pharmaceuticals, Alexandria, Egypt. ) was then injected. If no pain was elicited on needling, the needle was withdrawn to the subcutaneous tissue layer but not out of the skin and then moved in into different directions to encounter the sensitive spot in the MTrP region as evident by inducing pain before injection. The injection site was then compressed using a cotton bud for local haemostasis. This was the technique used in the control group A, while the intervention group B underwent the same technique except that the triggrt point was injected with $\sim 0.2$ $\mathrm{ml}$ dexamethasone $8 \mathrm{mg}+\sim 0.2 \mathrm{ml} 3 \%$ mepivicaine local anaesthetic. 
All the patients were instructed to avoid wide mouth opening, hard food chewing and stressful situations. Cetal (Cetal: paracetmol 500mg (micronized) tablets, Egyptian International Pharmaceutical Industries Co., Cairo, Egypt) was prescribed as pain killer only when needed during the first week, each patient received 3 treatment sessions at an interval of 2 days. In the second week, each patient received 2 treatment sessions at an interval of 3 days. At the end of the third week each patient received one treatment session. For both groups, the evaluation was realized in three moments: preoperatively (T1) as well as one week (T2) and 12 weeks (T3) after the end of the treatment sessions. The primary outcomes were the patients' current and worst pain intensity. The secondary outcome was the Maximum mouth opening (MMO).

VAS, ranging from 0 to 10 where $1-3$ is considered mild pain, 4-6 moderate, and 7-10 severe, was used for measurement of patients' current and worst pain intensity. The patients' worst pain is the worst pain experienced in the preceding 24 hours.

Statistical analysis of the results of this study was performed using SPSS (Statistical package for the social sciences- IBM ${ }^{\circledR}$ SPSS ${ }^{\circledR}$ Statistics Version 20 for Windows, IBM Corp., Armonk, NY, USA). Quantitative data were represented as mean \pm standard deviation, and Mann-Whitney U test was used to compare variables between the two groups. Qualitative data were represented as percentage or frequency, and Fisher's exact test was used to compare variables between the two groups. The results were considered statistically significant if the $p$ value was $\leq 0.05$.

\section{RESULTS}

The results of the 3 testing intervals pre-operative (T1), T 21 week post start of treatment and T3 12 weeks post operative were collected. 3 cases of group A failed to recall at the T3 stage and 2 cases of the group B also failed to comply to the follow up, so they were rolled out of the statistical analysis of the results.

Assessing the primary outcome of our study (pain intensity) at the proposed intervals of immediately pre operative T1 (base line score), 1week T2 and 12 weeks T3 were described in table 1. From the results of the visual analog scale (VAS) it has been apparent that in T1 both groups showed similar scores with statistically non significant difference between patients of both groups. $\mathrm{T} 2$ results showed an overall improvement in the pain intensity of both groups patients despite the slightly better results of pain relief described by patients of group B, but also statistically non significant difference between patients of both groups at this stage. T3 results showed a marked improvement in the pain score for both groups, yet slight lead to group B but with no statistical significant difference.

TABLE (1) Showing the summarization of the demographic characteristics of both groups, with statistical non significant differences in the age, sex and even duration of symptoms in the patients enrolled in both testing groups.

\begin{tabular}{|l|c|c|c|c|c|}
\hline \multirow{2}{*}{ Table 1 } & \multicolumn{2}{|c|}{ Group A } & \multicolumn{2}{c|}{ Group B } & p-value \\
\cline { 2 - 6 } & Mean & St Dev & Mean & St Dev & \\
\hline Patient Age (Years) & 26.3 & 2.06 & 28.7 & 4.35 & 0.956 \\
\hline Duration (weeks) of symptoms & 13 & 5.4 & 10 & 6.3 & 1.358 \\
\hline \multirow{2}{*}{ Gender } & Males & Females & Males & Females & \\
\cline { 2 - 7 } & 4 & 11 & 3 & 12 & \\
\hline
\end{tabular}


The results of the secondary outcome assessing the maximum mouth opening of both groups described in table 3, where the average MMO of the patients of both groups in $\mathrm{T} 1$ analysis showed statistically nonsignificant difference between patients of both groups. T2 showed a marked improvement in the range of mouth opening after 1 week of the injection therapy with slight better range in group B patients but with no significant difference than group A results. 12 weeks analysis showed a continued increase of the MMO in both groups but still with a statistically non significant advantage of any of the groups.

TABLE (2)

\begin{tabular}{|c|c|c|c|c|c|}
\hline VAS results & \multicolumn{2}{|c|}{ L.A. Group A } & \multicolumn{2}{|c|}{$\begin{array}{c}\text { Corticosteroid } \\
\text { Group B }\end{array}$} & \multicolumn{1}{|c|}{} \\
\cline { 2 - 6 } & Mean & St Dev & Mean & St Dev & P value \\
\hline T1 & 5.2 & 2.05 & 5.4 & 2.08 & 0.795 \\
\hline T2 (1 week) & 4.1 & 1.4 & 3.9 & 1.06 & 0.66 \\
\hline T3 (12 weeks) & 2,86 & 1.3 & 2.06 & 1.5 & 0.13 \\
\hline
\end{tabular}

TABLE (3)

\begin{tabular}{|c|c|c|c|c|c|}
\hline \multirow{2}{*}{$\begin{array}{c}\text { MMO in } \\
\text { mms }\end{array}$} & \multicolumn{2}{|c|}{ Group A } & \multicolumn{2}{c|}{ Group B } & \multirow{2}{*}{$\begin{array}{c}\text { P } \\
\text { value }\end{array}$} \\
\cline { 2 - 5 } & Mean & St Dev & Mean & St Dev & \\
\hline $\begin{array}{c}\text { Preoperative } \\
\text { operative T1 }\end{array}$ & 27.7 & 2.08 & 26.5 & 2.12 & 0.137 \\
\hline T2 ( 1 week) & 37.7 & 2.92 & 38.5 & 3.42 & 0.130 \\
\hline $\begin{array}{c}\text { T3 (12 } \\
\text { weeks) }\end{array}$ & 41.2 & 2.09 & 44.07 & 1.55 & 3.591 \\
\hline
\end{tabular}

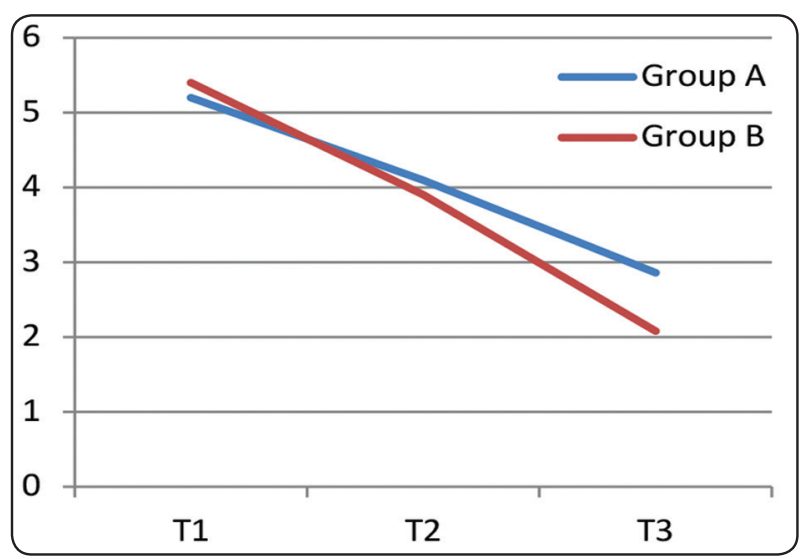

Chart 1 showing the flow of patients pain improvement along the follow up intervals of both groups.

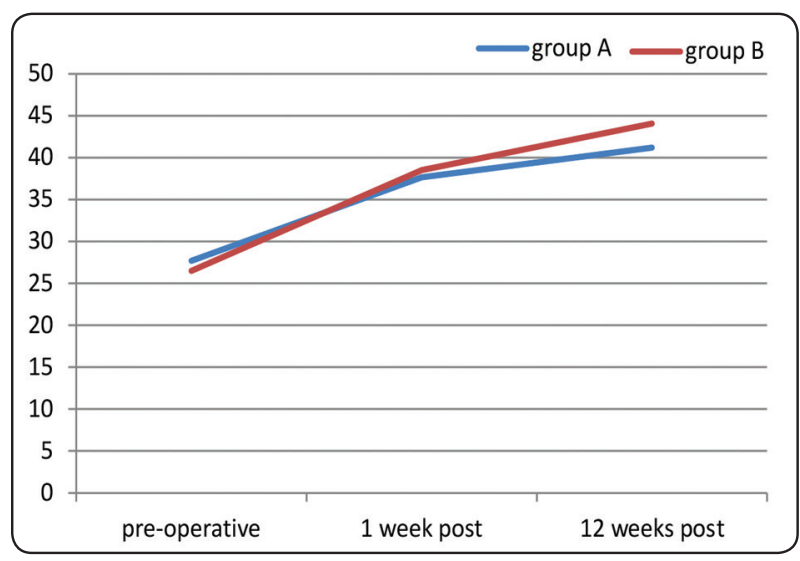

Chart 2 showing the flow of improvement of the MMO in both groups.

\section{DISCUSSION}

Myofascial pain is a highly prevalent disease that usually accompanied with the presence of myofascial trigger points, which are often the focus of diagnosis and treatment. The treatment aims to achieved pain relief and reestablish normal mandibular functions. Among these modalities used were dry needling and local anaesthesia injections. However the outcomes of the reported comparative studies between these modalities are contradictory and mandated further investigation. As it has been reported that local anaesthesia is a reliable needling drug for the management of MTrPs. But it is not clearly documented whether the association with a corticosteroid would show even better outcomes, 
specifically in the chronic cases. ${ }^{(14,17,20)}$ The aim of the present study is to assess and compare the effect of LA injections and LA with corticosteroids on deactivating MTrPs symptoms (pain and MMO) within the jaw muscles.

Injected solution was thought to dilute any nerve-sensitizing substances thereby reduce irritability and inactivate feedback mechanisms, as local anaesthetics has a local vasodilating influence that increases circulation at the trigger point area, accelerate metabolites removal and providing a local energy source, so results in limiting high frequency impulse discharge by the nerve. Depending on the local anesthetic agent injection site necrosis may destroy the trigger point. ${ }^{(17,18)}$

The frequency of needling therapies of active MTrPs used different protocols from single to repetitive needling within a brief course of treatment. The reported frequency of needling is utterly arbitrary. ${ }^{(21)}$ Due to the lack of standard treatment protocol, the current study applied repetitive needling along 3 weeks, three sessions in the $1^{\text {st }}$ week, two sessions in the $2^{\text {nd }}$ week and one session in the $3^{\text {rd }}$ week.

The primary outcome of interest was the change in pain from baseline to endpoint according to the visual analog scale. Patients of both groups started the study with nearly similar VAS scores and that was evident by the calculated $\mathrm{p}$ value to compare the results of both groups at that interval which was 0.795 , thus denoting statistically non significant difference between both study groups. While at the $1^{\text {st }}$ week follow up interval a marked relief in the pain was recorded from both groups but with a slight lead for the corticosteroid group over LA but without any statistical backup. At the endpoint of our study a considerable amount of patients of both groups reported pain free however other samples still reported pain scores. Corticosteroid group showed better overall results in both pain relief and reestablishment of the mandibular range of motion over the LA group despite of the statistically non significant analysis of the collected data of both groups at 12 weeks post operative proved by $\mathrm{p}$ value of 0.13 .
The assessment of the secondary outcome results of our study revealed that the start point for both groups was similar proofed by the statistically calculated $\mathrm{p}$ value of the T1 0.137 denoting statistical in significance, while in the T2 analysis it was evident the marked improvement in the range of mandibular functions with lead in the corticoid group over the LA group, but remaining of no statistical difference. The T3 results terminated our testing by an observed improvement in the MMO but also resulting in a calculated $P$ value of 3.591 which terminated our study on statistical insignificant difference in the final outcome of both groups.

The formation of a trigger point can be mediated through the CNS. As the injection modality results in an interruption in the pain transmission, so it obstructs the vicious cycle caused by the trigger point, thereby hampering the sensitivity and further development of new painful triggers. ${ }^{(15,16,21,22)}$

The results of our study came in accordance with Roberta de Abreu Venâncio et al. ${ }^{(3)}$ which stated that the use of local anesthetic agents in association with corticoids resulted in hampering the effects of central and peripheral sensitization thereby making the procedure painless and easing the post-operative period.

The use of corticosteroid injections has been documented in several studies to be a useful adjunct to the treatment modalities employed in managing musculoskeletal trigger areas, this came with accordance to our postulations in the current study. And it has been also stated that an injection protocol is at most effectiveness when adjunct with other pharmacologic and rehabilitation measures. ${ }^{(13,23)}$

\section{CONCLUSIONS}

Local anaesthesia injection alone and in combination with corticosteroids is an efficient method for the management of myofascial pain with active trigger points.

Corticosteroid adjunction with local anaesthesia resulted in better pain relief and MMO results than the use of LA alone for the management of MTrPs. 


\section{REFRENCES}

1. Alvarez JD and Rockwell PG. Trigger points: diagnosis and management. Am Fam Phys. 65(4):653-660, 2002.

2. Porta MA: Treatment of myofascial pain by injection with botulinum toxin. Rationale, procedure and outcomes. Adelphi Communications Ltda., Bollington, Macclesfield, Cheshire SK10 5JB, UK, 20 p.

3. Roberta de Abreu Venâncio, Francisco Guedes Pereira Alencar, Jr and Camila Zamperini Different Substances and Dry-Needling Injections in Patients with Myofascial Pain and Headaches. The Journal Of Craniomandibular Practice. 26, (2), 2008.

4. Thien Phu Do, Gerda Ferja Heldarskard, Lærke Tørring Kolding. tension-type headache. J Headache Pain. 19(1); $84,2018$.

5. Nagwan A Elsayed, Marwah Anas El-Wegoud, Omniya M Abdel Aziz , Ashraf F Nabhan and Emad S Helmy. Trigger Point Deactivation in Muscles of Mastication in Myofascial Pain Dysfunction (MPD) Patients: A Qualitative Systematic Review. Res Rep Oral Maxillofac Surg. 2;009, 2018.

6. Simons DG, Travell JG SL Facial muscles. In: Travell and Simons' Myofascial Pain and Dysfunction: The Trigger Point Manual. Vol. 1 Upper Half of the Body. (2nd edn), Lippincott, Williams \& Wilkins, USA. 417-418, 1999.

7. Borg-Stein J. Treatment of fibromyalgia, myofascial pain and related disorders. Phys Med Rehabil Clin N Am. 17: 491-510, 2006.

8. Atef F. Comparison between four treatment modalities for active myofascial triggers points. Plastic and Aesthetic Research. 1: 21-28, 2014.

9. Han SC, Harrison P: Myofascial pain syndrome and trigger-point management. Reg Anesth. 22(1); 89-101, 1997.

10. Hammi C, Schroeder JD, Yeung B. Trigger Point Injection. [Updated 2019 Dec 3]. In: StatPearls [Internet]. Treasure Island (FL): StatPearls Publishing; 2019 Jan-. Available from: https://www.ncbi.nlm.nih.gov/books/NBK542196/. (2019).

11. Renato Oliveira Ferreira da Silva, Paulo César Rodrigues Conti, Carlos dos Reis Pereira Araújo and Rafael dos Santos Silva. Evaluation of dry needling and $0.5 \%$ lidocaine injection therapies in myofascial pain trigger points in masticatory muscles. Dental Press J. Orthod. 17 (2), 2012.
12. Luis M. Gonzalez-Perez, Pedro Infante-Cossio, Mercedes Granados-Nuñez, and Francisco J. Urresti-Lopez. Treatment of temporomandibular myofascial pain with deep dry needling. Med Oral Patol Oral Cir Bucal. 17(5); 781-785, 2012.

13. Jess D Salinas and Elizabeth A Moberg-Wolff. Corticosteroid Injections of Joints and Soft Tissues. Physical Medicine and Rehabilitation. March 2019.

14. Raphael KG, et al.: Complementary and alternative therapy use by patients with myofascial temporomandibular disorders. J Orofac Pain. 17:36-41,2003.

15. Alvarez JD, Rockwell PG: Trigger points: diagnosis and management. Am Fam Phys. 65(4):653-660, 2002.

16. Porta MA. comparative trial of botulinum toxin type A and methylprednisolone for the treatment of myofascial pain syndrome and pain from chronic muscle spasm. Pain. 85:101-105,2000.

17. Tough EA, White AR, Cummings TM, Richards SH, Campbell JL. Acupuncture and dry needling in the management of myofascial trigger point pain: a systematic review and meta-analysis of randomised controlled trials. Eur J Pain. 13 (1):3-10, 2009.

18. Kietrys DM, Palombaro KM, Azzaretto E, Hubler R, Schaller B, Schlussel JM, et al. Effectiveness of dry needling for upper-quarter myofascial pain: a systematic review and meta-analysis. J Orthop Sports Phys Ther. 43 (9):620-34. 2013.

19. Donnelly JM, Fernández-de-las-Peñas C, Finnegan M, Freeman JL, eds. Travell, Simons \& Simons' Myofascial Pain and Dysfunction: The Trigger Point Manual. 3rd ed. Philadelphia: Wolters Kluwer; 2019.

20. Ge HY, Nie H, Madeleine P, Danneskiold-Samsøe B, Graven-Nielsen T, Arendt-Nielsen L. Contribution of the local and referred pain from active myofascial trigger points in fibromyalgia syndrome. Pain.15. 147 (1-3):233-40. 2009.

21. Lucas N, Macaskill P, Irwig L, Moran R, Bogduk N. Reliability of physical examination for diagnosis of myofascial trigger points: a systematic review of the literature. Clin J Pain. 25 (1):80-9. 2009.

22. Tough EA, White AR, Richards SH, Campbell JL. Myofascial trigger point needling for whiplash associated pain- a feasibility study. Man Ther. 15 (6):529-35. 2010

23. Richardson SS, Schairer WW, Sculco TP, Sculco PK. Comparison of Infection Risk with Corticosteroid or Hyaluronic Acid Injection Prior to Total Knee Arthroplasty. J Bone Joint Surg Am. 16. 101 (2):112-8. 2019. 